\title{
The role of microRNAs in osteoporosis: A brief review
}

\author{
Despina Misiaka', George I. Lambrou ${ }^{1,2,3}$ \\ 'Postgraduate Program “Metabolic Bones Diseases", National and Kapodistrian University of Athens, Medical School, Athens, \\ Greece; \\ ¿Laboratory for the Research of the Musculoskeletal System "Th. Garofalidis", National and Kapodistrian University of Athens, \\ Medical School, Athens, Greece; \\ ${ }^{3}$ Choremeio Research Laboratory, First Department of Pediatrics, School of Medicine, National and Kapodistrian University of \\ Athens, Athens, Greece, Athens, Greece
}

\begin{abstract}
Osteoporosis is a common disease, characterized by loss of bone mass and increased incidence of fractures, due to increased bone resorption through osteoclasts and decreased bone production through osteoblasts. MicroRNA (miRNA) is a type of short non-coding RNA with a length of 20-25 nucleotides, which exerts post-transcriptional control by inhibiting or degrading target genes. Recently, it has been noticed that miRNAs may play a significant role in the pathophysiology of osteoporosis as they influence the process of bone remodeling by regulating the differentiation and function of osteoblasts and osteoclasts. More specifically, miRNAs promote apoptosis and inhibit the proliferation and differentiation of osteoblasts. At the same time, there are miRNAs that promote osteoclast apoptosis, thus delaying bone loss in patients with osteoporosis, while also affecting the osteogenetic differentiation of mesenchymal stem cells. MiRNAs-based treatments for osteoporosis have not yet been in the clinical stage, so more studies are needed.
\end{abstract}

Keywords: microRNA, Osteoblasts, Osteoclasts, Osteoporosis

\section{Introduction}

Osteoporosis is a high frequency disease with global impact, characterized by loss of bone mass, disturbance of bone microarchitecture, increased bone fragility and high risk of fractures ${ }^{1}$. The main pathophysiologic mechanism of osteoporosis is increased bone resorption caused by osteoclast activity and decreased bone production caused by osteoblast activity ${ }^{2}$. As bone cells respond to mechanical stimuli, bone tissue is maintained through a feedback system. In particular, changes in the local mechanical environment trigger bone cells to modify bone structure in order to meet the new requirements. Mechanical unloading causes significant bone loss due to attenuation of bone formation, while mechanical loading leading to high stresses increases bone formation. These changes ultimately affect bone formation and absorption by affecting the balance between osteoblast and osteoclast activity.

Osteoporosis is classified into two types, type I and type II. In type I (postmenopausal osteoporosis), bone loss is mainly associated with increased osteoclastic activity, leading to increased bone resorption and bone metabolism and causing bone loss and bone destruction. In type II osteoporosis, bone loss is mainly related to the function of osteoblasts which is disrupted, reducing bone production, while the function of osteoclasts is generally normal ${ }^{3}$.

MicroRNA is a type of short non-coding RNA with a length of 20-25 nucleotides, which exerts post-transcriptional control by inhibiting or degrading target genes. Mature miRNAs have complementary sites in the 3'-untranslated region (UTR) of the target mRNA and combine with each other so that translation cannot occur, thereby inhibiting

The authors have no conflict of interest.

Corresponding author: George I. Lambrou, First Department of Paediatrics, National and Kapodistrian University of Athens, Choremeio Research Laboratory, Thivon \& Levadeias 8, 11527 , Goudi, Athens, Greece

E-mail: glamprou@med.uoa.gr

Edited by: Konstantinos Stathopoulos

Accepted 5 May 2021 
gene expression. The miRNAs recognize the target mRNA through a sequence of 2-8 nucleotides that carry them at their 5'-end and is called a "seed sequence". Each miRNA may regulate the expression of a plethora of genes and each gene is regulated by multiple miRNAs. Through posttranscriptional gene silencing, miRNAs play an important regulatory role in cell proliferation, differentiation and apoptosis, tumor progression and development, and other physiological and pathological processes ${ }^{4}$.

MiRNAs appear to act in two different ways on target mRNAs, either by inhibiting ribosome translation or by inducing mRNA cleavage. The pathway by which miRNA will act on the target mRNA depends on: 1) the number of nucleotide sequence motifs recognizing its 5'-end, 2) accessibility at the binding site, and 3 ) the degree of complementarity with the binding site ${ }^{5}$. When the miRNA is almost completely combined with the mRNA encoding the proteins, the miRNA and mRNA transcripts form the miRISC silencing complex (miRNA-associated polyprotein RNA-induced silencing complex) and the mRNA in the target genes is further degraded by nuclease. However, miRNAs in most mammals do not degrade the target mRNA, but regulate gene expression through another mechanism. Partial complementarity between the mRNA and regions beyond the 5'-end miRNA stabilizes the binding between miRNA and mRNA. These miRNAs form RISC-like complexes through incomplete base pairing and 3'- UTRs, which inhibit gene translation at the post-transcriptional level and reduce the protein expression level of its target genes.

\section{The role of microRNAs in Osteoporosis}

Bones are in a constant state of remodeling throughout the life of the human body, maintaining a dynamic balance. This process mainly involves bone resorption by osteoclasts and bone formation by osteoblasts. Hematopoietic stem cells eventually form osteoclasts through osteoclasts precursor to absorb bone matrix and minerals. Monocytes prepare the bone resorption surface for osteoblasts and then the osteoblasts secrete bone matrix, followed by mineralization and differentiation of some osteoblasts into bone cells, completing the bone remodeling process ${ }^{6}$.

Literature data has reported that miRNAs play an important regulatory role in the process of bone remodeling, especially by regulating the differentiation and function of osteoblasts and osteoclasts and affecting the development and progression of osteoporosis and other bone diseases ${ }^{7-9}$. Recently, research has focused on the mechanism of action of miRNAs in the development and progression of osteoporosis, in an attempt to find an effective gene therapy to treat or delay the disease.

\section{The role of miRNAs in osteoblasts}

The process of osteogenesis includes 4 stages: proliferation of osteoblasts, maturation and mineralization of the extracellular matrix and apoptosis of osteoblasts. Many factors can act at these stages, regulating bone production ${ }^{10}$. Osteoblasts are the main functional cells of bone formation, responsible for the production, secretion and mineralization of bone tissue. Bones are constantly reshaping and this process involves the attachment of osteoclasts to the area of bone resorption, the secretion of acid-dissolving minerals, and the secretion of proteases for the degradation of bone matrix. The osteoblasts then migrate to the site of absorption and secrete bone matrix which then is mineralized and forms new bone. The balance between bone resorption and bone production is the key to maintaining normal bone mass ${ }^{11}$.

As the body ages, the balance between bone production and bone resorption is disturbed in both men and women due to the reduction in the number and activity of osteoblasts, leading to osteoporosis. The external mechanism of this process may be age-dependent changes in the microenvironment of bone tissue, such as hormone levels and growth factors. The internal mechanism mainly involves osteoblasts senescence ${ }^{12}$. Recently, an increasing number of studies have noticed that the regulatory role of miRNAs must be taken into consideration in the process of proliferation, differentiation and apoptosis of osteoblasts.

Previous research has shown that some miRNAs are sensitive to mechanical stimuli, which could regulate osteoblast function. For example, some miRNAs are involved in pro-osteoblast differentiation, such as miR-20a, miR21, miR-19b, miR-34a, miR-34c, miR-140, and miR$200 b^{13}$. MiR-103 is upregulated in response to osteoblast differentiation induced by cyclic mechanical extension and significantly inhibits osteoblast differentiation ${ }^{14}$. In addition, miR-153 is sensitive to mechanical loading and regulates osteoblast differentiation by directly targeting BMPR $2^{15}$. MiR-33-5p is a new mechanically sensitive miRNA that can promote the differentiation of osteoblasts and participate in the regulation of differentiation caused by changes in the mechanical environment, suggesting this miRNA as a potential target for the treatment of abnormal bone loss. These results suggest that miRNAs may play an important role in the detection of mechanical loads by osteoblasts.

Oxidative stress is a known factor in the pathogenesis of osteoporosis, as uncontrolled excess of free radicals may destroy bone production and cause loss of bone mass. The FoxO family plays a very important role in protecting cells from oxidative stress and FoxO 1 is particularly critical in bone tissue. Activation of FoxO1 may promote osteoblast proliferation and differentiation and inhibit osteoblast apoptosis. Kim et al. (2012) found that miR-182 in patients with osteoporosis is significantly increased in comparison to the normal population. Elevated miR-182 inhibits the expression of FoxO1, thus eliminating the protective effect of oxidative stress, promoting osteoblast apoptosis and inhibiting the proliferation and differentiation of osteoblasts $^{16}$.

Removal of Dicer factor from animal models (mice) appears to affect bone growth after birth as well as the 
amount of bone mass through the effect of miRNA deficiency in the proteins of the extracellular matrix ${ }^{17}$. In particular, research has shown a significant increase in bone mass in both cancellous and cortical bones between 4 and 8 weeks after birth, an increase that continued for at least 8 months. Osteoblast activity was also accelerated in the extracellular matrix where an increase in collagen-related protein expression was observed. Phenotypically increased bone mass (BMD was normal) was found to be directly correlated with the loss of function of miRNAs in mature osteoblasts and no correlation with inhibition of bone resorption was observed ${ }^{17}$.

It is important to note that studies performed on animal models of genetically modified mice after the elimination of Dicer have revealed the existence of a specific group of miRNAs called "osteomiRs", which may be responsible for producing phenotypes, which are characterized by increased bone mass. "OsteomiRs" begin to be expressed already at the stage of osteoprogenic cell with a gradual increase in their concentration during the course of osteoblastogenesis ${ }^{18}$. The target of "osteomiRs" is proteins of the extracellular matrix, transcription factors and factors that affect osteoblastogenesis. A typical example of this group of miRNAs, which regulate osteogenesis, is miR-29, which appears to have multiple and distinct activities at all levels of osteoblastogenesis. Initially, it suppresses known inhibitors of osteoblastogenesis in the osteoprogenic cell stage, such as HDAC4, which reduces the transcriptional activity of the MAPK5 protein, and DUSP2-specific phosphatase, which inactivates E1O by phosphorylation. Then it suppresses the expression of Dickoff 1 factor (DKK 1), which is the major inhibitor of the Wnt signaling pathway, accelerating the maturation of the early osteoblast into a mature osteoblast ${ }^{19}$. In addition to osteoblastogenesisregulating proteins, miR-29 also targets bone structure. MiR-29 acts on type I collagen, which is a building block of at least $90 \%$ of the organic matrix. At least 5 other groups of miRNAs, target collagen proteins (for example miR-196, let-7 and miR 15/16) and their expression increases during the mineralization stage of the extracellular matrix ${ }^{18}$. Finally, the SPARC protein, also known as osteonectin, a structural component of the extracellular matrix, is the target for miR$29^{20}$. Wang et al. (2013) found that miR-214 expression in femoral tissues in adults of different age groups increased with age and was negatively correlated with BGLAP and ALP associated with bone production ${ }^{21}$. In animal models, miR214 was strongly expressed in osteoblasts of mice that underwent bilateral ovariectomy, suggesting that miR-214 may inhibit osteoblast activity and mineralization of the extracellular matrix.

A study by Inose et al. (2009) concluded that increased regulation of miR-206 in osteoblasts in patients with osteoporosis inhibits Cx43 protein expression, thereby affecting osteoblast function ${ }^{22}$. In addition, miR-218 enhances Wnt activity and regulates osteoblast genes ${ }^{23}$.
MiR-210 improves postmenopausal osteoporosis due to estrogen deficiency by promoting VEGF expression and osteoblast differentiation ${ }^{24}$. These findings demonstrate that miRNAs significantly affect osteoblast differentiation.

\section{The role of miRNAs in osteoclasts}

Osteoclasts are composed of multinucleated giant cells, which are distributed mainly on the surface of the bone. Prior to bone resorption, osteoclast precursors differentiate into mature osteoclasts, which attach to the bone surface creating an isolated microenvironment, thus degrading the extracellular matrix of bone tissue ${ }^{25}$. Many studies have confirmed the regulatory role of miRNAs in the process of bone resorption by osteoclasts. Some miRNAs play a role in the evolution of osteoclast differentiation, proliferation and activity, thus influencing the development of osteoporosis. Some miRNAs can promote osteoclast apoptosis, thus delaying bone loss in patients with osteoporosis.

The miRNAs undergo a process from precursor miRNAs to mature miRNAs prior to performing post-transcriptional control on the target genes. A small number of miRNAs are known to contribute to osteoclastogenesis by affecting mainly the precursor stages of the process. First of all, miR223 was found to enhance the reduction of NFIA (an inhibitor of osteoclastogenesis). The decrease in NFIA causes an increase in MCSF, which in turn leads to an increase in RANKL receptor production by precursor osteoclasts, accelerating their maturation ${ }^{26}$. A second molecular event regarding the regulation of osteoclastogenesis has been revealed by research on miR-155 where it appears to be involved in the activity of two different cell lines. Specifically, miR-155 induces the differentiation of hematopoietic stem cells into macrophages while at the same time, by inhibiting the factor MIFT (catalytic factor for the differentiation of precursor osteoclasts), it blocks the maturation of osteoclasts $^{26}$. Therefore, miR-155 negatively regulates the maturation of osteoclasts while at the same time it regulates the maturation of hematopoietic stem cells in macrophages as a molecular "switch". In addition to the early stages of osteoclast maturation, which are affected by miR-155 and miR-223, miR-21 was found to be involved in the final stage of osteoclast differentiation. The expression of miR21 increases in the final stages of osteoclast differentiation due to the increase in RANKL factor. In turn, miR-2 1 induces the expression of c-Fos factor, which is a key regulator of osteoclastogenesis. Increase in c-fos factor in mature osteoclasts in turn inhibits miR-21 expression, creating an one-way negative feedback mechanism ${ }^{27}$.

Recently, Wang et al. (2014) found that osteoclast survival rates in miR-9 and miR-181a-knockout mice were higher than in wild-type mice ${ }^{28}$. Cbl protein is an important E3 ubiquitin ligase involved in bone resorption, which has been widely recognized as the target gene for miR-9 and miR-181a. Cbl can regulate osteoclast apoptosis by promoting the degradation of the ubiquitin-dependent Bim 
pre-apoptotic gene. Bim protein is a member of the BCL-2 family of proteins and the Bim gene has been identified as an important pre-apoptotic gene of osteoclasts.

Bloodmonocytes, as precursors of osteoclasts, can secrete a number of cytokines that regulate osteoclastogenesis, such as IL-1, IL-6, and TNF-a. Therefore, monocytes have been studied in osteoporosis in recent years. Cao et al. (2014) found that miR-422a is significantly elevated in postmenopausal patients with osteoporosis ${ }^{29}$.

A new topic of research is the possible action of miRNAs through cell-to-cell communication. In this case, cells that are in contact via the ligand receptor complex, such as the RANKL-RANK complex between osteoblast and osteoclast, appear to be able to exchange mature miRNAs using exosomes. This will result in the same miRNA regulating protein expression even in different cell lines ${ }^{30}$.

In conclusion, in vivo studies show that disturbances in the biosynthesis of miRNAs through the lack of Dicer factor in bone affect osteogenesis at various stages. MiRNAs play an important role in the maturation and proliferation of osteoblasts, osteoclasts and osteocytes in each of the three bone cell lines. Examples of miRNAs have already been reported, which target different proteins at distinct stages of bone cell maturation, essentially playing the role of orchestrator in bone formation.

\section{The role of miRNAs in mesenchymal stem cells}

Mesenchymal stem cells are a group of stem cells with the ability to self-renew and multiply. In different inductive environments, they can differentiate into osteoblasts, adipocytes, chondroblasts and neuroblasts. Mesenchymal stem cells are progenitor cells of osteoblasts and osteocytes. Controlling the differentiation of mesenchymal stem cells into osteoblasts is vital to the management of bone loss. Some key molecules or signaling pathways that play a role in promoting osteogenic differentiation of mesenchymal stem cells are bone morphogenetic proteins (BMPs), Runx2, alkaline phosphatase (ALP), and the Wnt signaling pathway. These molecules or pathways interact with each other, forming a huge regulatory network ${ }^{31}$. MiR-29a, miR-218 and mir335-5p upregulate osteoblast differentiation by affecting the Dickkopf molecule of the Wnt signaling pathway miR-2861.

Different species, different target cells, and different regulation of target genes are likely to cause some differences in the functions of the same miRNA. For example, high expression miR-2 10 may promote osteogenic differentiation of bone marrow-mesenchymal stem cells in mice. In contrast, miR-210 is reduced in osteogenic differentiation of human mesenchymal cells and the inhibition of miR-210 may promote osteogenic differentiation of human mesenchymal stem cells.

Several miRNAs have been identified to be involved in osteogenesis or lipogenesis. Li et al. (2015) found that
miR-188 was highly expressed in elderly mice and humans and that it regulates the branching differentiation of bone marrow mesenchymal stem cells into osteoblasts and adipocytes $^{32}$. miR-204 and miR-211 have been reported to act as vital negative regulators of Runx 2 to promote lipogenesis and suppress osteogenesis in bone marrow mesenchymal stem cells ${ }^{33}$. MiR-205 has been shown to have adverse effects on osteogenic differentiation of bone marrow mesenchymal stem cells, while miR-2 1 promoted osteogenic differentiation of mesenchymal stem cells via the $\mathrm{PI} 3 \mathrm{~K} / \mathrm{\beta}$-catenin pathway ${ }^{34}$.

MiR-23a and miR-23b were found to be significantly reduced in bone marrow mesenchymal stem cells of elderly mice and humans. Overexpression of miR-23a and miR23b in bone marrow mesenchymal stem cells promoted osteogenic differentiation, while inhibition of miR-23a/miR23b increased adipogenic differentiation. Transmembrane protein 64 (Tmem64), which has expression levels inversely related to those of miR-23a / miR-23b in elderly and young mice, was identified as the primary target of $\mathrm{miR}-23 \mathrm{a} / \mathrm{miR}$ $23 \mathrm{~b}$ in the differentiation of bone marrow mesenchymal stem cells. Researchers concluded that miR-23a / miR-23b plays a critical role in regulating mesenchymal line differentiation through suppression of Tmem6 $4^{35}$. Mesenchymal stem cells can not only differentiate into osteoblasts, but also modulate osteoclastogenesis through the expression of RANKL and osteoprotegerin. Stem mesenchymal cells can express RANKL to promote osteoclastogenesis and also express osteoprotegerin receptors and secrete osteoprotegerin to inhibit osteoclastogenesis ${ }^{36,37}$.

MiR-133 and miR-135 functionally inhibit osteogenic differentiation by reducing the Runx 2 and Smad5 pathways, which contribute synergistically to bone formation ${ }^{38}$. Runx 2 is an osteoblast-specific gene that has been widely studied as a key molecule in the pathogenesis of osteoporosis. MiR23a, miR-30, miR-133, miR-135a, miR-137, miR-204, miR-205, miR-211, miR-217, miR-335, miR-338 and miR-433 downregulate osteoblast differentiation through Runx2 regulation. $\mathrm{Li}$ et al. discovered a new miRNA (miR2861) that can reduce the degradation of Runx2 and promote osteogenetic differentiation of mesenchymal stem cells in mice by inhibiting HDAC5 expression ${ }^{39}$. MiR-2861 expression has also been found to be reduced in patients with postmenopausal osteoporosis.

Many inflammatory factors, such as tumor necrosis factor (TNF- $a$ ), have been shown to inhibit osteogenetic differentiation of mesenchymal stem cells in estrogendeficient secondary osteoporosis. Yang et al. (2013) found that miR-21 expression is significantly lower in mesenchymal stem cells in mice that underwent bilateral ovariectomy ${ }^{40}$. Subsequent experiments have shown that the decreased expression of miR-21 is due to inhibition of TNF-a. MiR-2 1 can promote osteogenetic differentiation of mesenchymal stem cells by inhibiting the expression of the Spry 1 target gene. Spry 1 is an inhibitor of the tyrosine 
kinase receptor (RTK) pathway that negatively regulates multiple RTK signaling pathways, such as the FGF and MAPK signaling pathways. Spry 1 regulates osteogenetic differentiation of mesenchymal stem cells, with a negative manner.

Eskildsen et al. (2011) discovered a type of miRNA, miR-138, which is significantly reduced during osteogenic differentiation of mesenchymal stem cells ${ }^{41}$. The researchers used anti-miR-138 to block the expression of miR-138, so as to promote bone formation, which could become an effective therapeutic strategy for osteoporosis in the future.

In animal model studies, the absence of mature miRNAs in both osteoclast mononuclear precursor cells and mature osteoclasts in genetically modified mice resulted in phenotypes with increased bone mass due to both reduced number and reduced activity of osteoclasts ${ }^{26,27,42}$. In addition, osteoclasts could not be produced by bone marrow hematopoietic cells in either cell group in the mouse models. Also, no osteoclasts were able to mature from bone marrow hematopoietic stem cells in which either the Drosha enzyme (miRNA transcription activation enzyme) or the RISC complex (mRNA responsible for binding to miRNAs) had previously been inactivated ${ }^{26}$. These studies confirm that the loss of miRNAs in osteoclast progenitor cells inhibits the process of osteoclastogenesis.

\section{Conclusions}

In normal cells, the expression of various miRNAs contributes to the maintenance of the balance of cell proliferation, differentiation, apoptosis and other normal processes. The same miRNA can have a variety of different target genes, so that disruption of miRNA expression can cause a number of serious intracellular dysfunctions ${ }^{43}$.

Research on miRNAs has focused on the growth and development of tumors, but emerging data have shown that miRNAs also play an important role in regulating the pathogenesis of various bone diseases. In particular, the association between miRNAs and the pathogenesis of osteoporosis has been investigated. It is worth noting, however, that the important role of miRNAs in regulating the differentiation and function of osteoblasts and osteoclasts is only the first step in clinical practice, and more studies are needed to successfully implement miRNA-based osteoporosis treatments.

Current studies have shown that tissue-specific overexpression or inhibition of specific miRNAs has some therapeutic effect in animal models, while some miRNAsbased therapies are in clinical trial ${ }^{44,45}$. MiRNAs-based treatments for osteoporosis have not yet been found clinically, so more studies are needed. In the future, it is expected, through overexpression or inhibition of miRNAs, to delay bone loss and promote bone reshaping as a way of treating osteoporosis.

\section{References}

1. Armas LA, Recker RR. Pathophysiology of osteoporosis: new mechanistic insights. Endocrinology and metabolism clinics of North America 2012;41(3):475-86.

2. Yedavally-Yellayi S, Ho AM, Patalinghug EM. Update on Osteoporosis. Prim Care 2019;46(1):175-90

3. Sozen T, Ozisik L, Basaran NC. An overview and management of osteoporosis. Eur J Rheumatol 2017;4(1):46-56.

4. Yates LA, Norbury CJ, Gilbert RJ. The long and short of microRNA. Cell 2013;153(3):516-9

5. Nilsen TW. Mechanisms of microRNA-mediated gene regulation in animal cells. Trends Genet 2007:23(5):243-9.

6. Datta HK, Ng WF, Walker JA, Tuck SP, Varanasi SS. The cell biology of bone metabolism. J Clin Pathol 2008;6 1 (5):577-87.

7. Bae Y, Yang T, Zeng HC, Campeau PM, Chen Y, Bertin T, et al. miRNA$34 \mathrm{C}$ regulates Notch signaling during bone development. Human molecular genetics 2012:21(13):2991-3000

8. Fu HL, Pan HX, Zhao B, Dong BC, Shao L, Fu GS, et al. MicroRNA-100 inhibits bone morphogenetic protein-induced osteoblast differentiation by targeting Smad1. Eur Rev Med Pharmacol Sci 2016:20(18):3911-9.

9. Liu J, Li Y, Luo M, Yuan Z, Liu J. MicroRNA-214 inhibits the osteogenic differentiation of human osteoblasts through the direct regulation of baculoviral IAP repeat-containing 7. Exp Cell Res 2017;351(2): 157-62.

10. DucyP,SchinkeT,KarsentyG. Theosteoblast:asophisticatedfibroblast under central surveillance. Science 2000;289(5484): 1501-4.

11. Hadjidakis DJ, Androulakis, II. Bone remodeling. Ann N Y Acad Sci 2006; 1092:385-96.

12. Marie PJ, Kassem M. Osteoblasts in osteoporosis: past, emerging, and future anabolic targets. European journal of endocrinology 2011;165(1):1-10.

13. Papaioannou G, Mirzamohammadi F, Kobayashi T. MicroRNAs involved in bone formation. Cellular and molecular life sciences: CMLS 2014;71(24):4747-61

14. Zuo B, Zhu J, Li J, Wang C, Zhao X, Cai G, et al. microRNA-103a functions as a mechanosensitive microRNA to inhibit bone formation through targeting Runx2. Journal of bone and mineral research: the official journal of the American Society for Bone and Mineral Research 2015;30(2):330-45.

15. Cao Y, LV Q, LV C. MicroRNA-153 suppresses the osteogenic differentiation of human mesenchymal stem cells by targeting bone morphogenetic protein receptor type II. Int J Mol Med 2015; 36(3):760-6.

16. Kim KM, Park SJ, Jung SH, Kim EJ, Jogeswar G, Ajita J, et al. miR182 is a negative regulator of osteoblast proliferation, differentiation, and skeletogenesis through targeting FoxO 1. Journal of bone and mineral research : the official journal of the American Society for Bone and Mineral Research 2012;27(8): 1669-79.

17. Gaur T, Hussain S, Mudhasani R, Parulkar I, Colby JL, Frederick D, et al. Dicer inactivation in osteoprogenitor cells compromises fetal survival and bone formation, while excision in differentiated osteoblasts increases bone mass in the adult mouse. Dev Biol 2010;340(1):10-21

18. Li Z, Hassan MQ, Jafferji M, Aqeilan RI, Garzon R, Croce CM, et al. Biological functions of miR-29b contribute to positive regulation of osteoblast differentiation. J Biol Chem 2009;284(23): 15676-84.

19. Ling HY, Wen GB, Feng SD, Tuo QH, Ou HS, Yao CH, et al. MicroRNA-375 promotes 3T3-L1 adipocyte differentiation through modulation of extracellular signal-regulated kinase signalling. Clin Exp Pharmacol Physiol 201 1;38(4):239-46. 
20. Kapinas K, Kessler CB, Delany AM. miR-29 suppression of osteonectin in osteoblasts: regulation during differentiation and by canonical Wnt signaling. J Cell Biochem 2009; 108(1):216-24.

21. Wang X, Guo B, Li Q, Peng J, Yang Z, Wang A, et al. miR-2 14 targets ATF4 to inhibit bone formation. Nat Med 2013;19(1):93-100.

22. Inose H, Ochi H, Kimura A, Fujita K, Xu R, Sato S, et al. A microRNA regulatory mechanism of osteoblast differentiation. Proceedings of the National Academy of Sciences of the United States of America 2009; 106(49):20794-9.

23. Hassan MQ, Maeda $Y$, Taipaleenmaki H, Zhang W, Jafferji M, Gordon JA, et al. miR-2 18 directs a Wnt signaling circuit to promote differentiation of osteoblasts and osteomimicry of metastatic cancer cells. J Biol Chem 2012;287(50):42084-92.

24. Liu XD, Cai F, Liu L, Zhang Y, Yang AL. MicroRNA-2 10 is involved in the regulation of postmenopausal osteoporosis through promotion of VEGF expression and osteoblast differentiation. Biol Chem 2015;396(4):339-47.

25. Teitelbaum SL. Bone resorption by osteoclasts. Science 2000; 289(5484): 1504-8

26. Sugatani T, Hruska KA. Impaired micro-RNA pathways diminish osteoclast differentiation and function. J Biol Chem 2009; 284(7):4667-78.

27. Sugatani T, Vacher J, Hruska KA. A microRNA expression signature of osteoclastogenesis. Blood. 2011;117(13):3648-57.

28. Wang S, Tang C, Zhang Q, Chen W. Reduced miR-9 and miR181 a expression down-regulates Bim concentration and promote osteoclasts survival. Int J Clin Exp Pathol 2014;7(5):2209-18.

29. Cao Z, Moore BT, Wang Y, Peng XH, Lappe JM, Recker RR, et al. MiR422a as a potential cellular microRNA biomarker for postmenopausal osteoporosis. PloS one 2014;9(5):e97098.

30. Gamez B, Rodriquez-Carballo E, Ventura F. MicroRNAs and posttranscriptional regulation of skeletal development. J Mol Endocrinol 2014;52(3):R179-97.

31. Bielby R, Jones E, McGonagle D. The role of mesenchymal stem cells in maintenance and repair of bone. Injury 2007;38 Suppl 1:S26-32.

32. Li CJ, Cheng P, Liang MK, Chen YS, Lu Q, Wang JY, et al. MicroRNA-188 regulates age-related switch between osteoblast and adipocyte differentiation. The Journal of clinical investigation 2015;125(4):1509-22.

33. Huang J, Zhao L, Xing L, Chen D. MicroRNA-204 regulates Runx2 protein expression and mesenchymal progenitor cell differentiation. Stem Cells 2010;28(2):357-64

34. Hu N, Feng C, Jiang Y, Miao Q, Liu H. Regulative Effect of Mir205 on Osteogenic Differentiation of Bone Mesenchymal Stem
Cells (BMSCs): Possible Role of SATB2/Runx2 and ERK/MAPK Pathway. International journal of molecular sciences 2015; 16(5): 10491-506.

35. Guo Q, Chen Y, Guo L, Jiang T, Lin Z. miR-23a/b regulates the balance between osteoblast and adipocyte differentiation in bone marrow mesenchymal stem cells. Bone Res 2016;4:16022.

36. Giuliani N, Bataille R, Mancini C, Lazzaretti M, Barille S. Myeloma cells induce imbalance in the osteoprotegerin/osteoprotegerin ligand system in the human bone marrow environment. Blood 2001;98(13):3527-33

37. Gori F, Hofbauer LC, Dunstan CR, Spelsberg TC, Khosla S, Riggs BL. The expression of osteoprotegerin and RANK ligand and the support of osteoclast formation by stromal-osteoblast lineage cells is developmentally regulated. Endocrinology 2000; 141(12):4768-76.

38. Li Z, Hassan MQ, Volinia S, van Wijnen AJ, Stein JL, Croce CM, et al. A microRNA signature for a BMP2-induced osteoblast lineage commitment program. Proceedings of the National Academy of Sciences of the United States of America 2008; 105(37): 1390611.

39. Li H, Xie H, Liu W, Hu R, Huang B, Tan YF, et al. A novel microRNA targeting HDAC5 regulates osteoblast differentiation in mice and contributes to primary osteoporosis in humans. The Journal of clinical investigation 2009; 1 19(12):3666-77.

40. Yang N, Wang G, Hu C, Shi Y, Liao L, Shi S, et al. Tumor necrosis factor alpha suppresses the mesenchymal stem cell osteogenesis promoter miR-2 1 in estrogen deficiency-induced osteoporosis. Journal of bone and mineral research: the official journal of the American Society for Bone and Mineral Research 2013;28(3):559-73.

41. Eskildsen T, Taipaleenmaki H, Stenvang J, Abdallah BM, Ditzel N, Nossent AY, et al. MicroRNA-1 38 regulates osteogenic differentiation of human stromal (mesenchymal) stem cells in vivo. Proceedings of the National Academy of Sciences of the United States of America 2011;108(15):6139-44.

42. Mizoguchi F, Izu Y, Hayata T, Hemmi H, Nakashima K, Nakamura T, et al. Osteoclast-specific Dicer gene deficiency suppresses osteoclastic bone resorption. J Cell Biochem 20 10; 109(5):866-75.

43. Kasinski AL, Slack FJ. Epigenetics and genetics. MicroRNAs en route to the clinic: progress in validating and targeting microRNAs for cancer therapy. Nat Rev Cancer 201 1; 1 1(12):849-64.

44. Kapinas K, Delany AM. MicroRNA biogenesis and regulation of bone remodeling. Arthritis Res Ther 2011;13(3):220.

45. Tiemann K, Rossi JJ. RNAi-based therapeutics-current status, challenges and prospects. EMBO Mol Med 2009; 1(3): 142-51. 